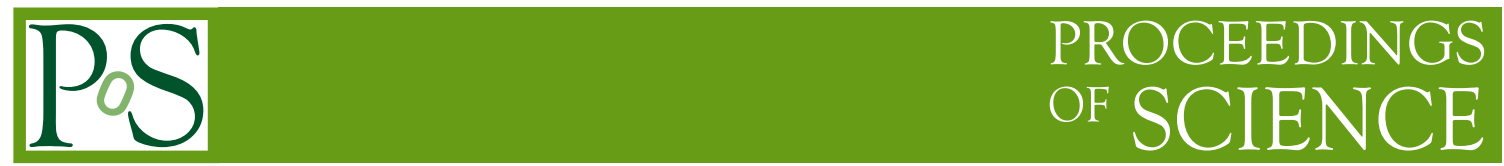

\title{
Measurements of CKM matrix elements
}

\author{
Fabio Ferrari $^{a, 1, *}$ \\ ${ }^{a}$ University of Bologna and INFN, \\ via Irnerio 46, Bologna, Italy \\ E-mail: fabio.ferrari@cern.ch
}

Precise theoretical predictions of the hadronic parameters related to exclusive semileptonic $b$ hadron decays allow for accurate determinations of the CKM matrix elements $\left|V_{\mathrm{cb}}\right|$ and $\left|V_{\mathrm{ub}}\right|$. The large production of $B_{s}^{0}$ mesons at the LHC allows LHCb to provide complementary information with respect to those from $B$-factories. In these proceedings, the latest $\mathrm{LHCb}$ results on the determination of $\left|V_{\mathrm{cb}}\right|$ with $B_{s}^{0} \rightarrow D_{s}^{(*)-} \mu^{+} v_{\mu}$ decays and on the measurement of the shape of the $B_{s}^{0} \rightarrow D_{s}^{*-} \mu^{+} v_{\mu}$ differential decay rate are presented.

40th International Conference on High Energy physics - ICHEP2020

July 28 - August 6, 2020

Prague, Czech Republic (virtual meeting)

\footnotetext{
${ }^{1}$ For the LHCb collaboration.

* Speaker
} 


\section{Introduction}

The coupling strength between $b$ and $c$ quarks, expressed by the element $\left|V_{\mathrm{cb}}\right|$ of the CabibboKobayashi-Maskawa (CKM) matrix, can be accessed in a clean way through studies of $\bar{b} \rightarrow \bar{c} l^{+} v_{l}$ transitions, where $l$ represents either an electron or a muon. So far, two complementary methods have been exploited to determine $\left|V_{\mathrm{cb}}\right|$. One approach measures the decay rate by inspecting at inclusive $b$-hadron decays to final states involving a $c$-flavored hadron and a charged lepton, while the other measures the rate of a specific (exclusive) decay. The average of the first method yields $\left|V_{\mathrm{cb}}\right|=(42.19 \pm 0.78) \times 10^{-3}$, while the second gives $\left|V_{\mathrm{cb}}\right|=(39.25 \pm 0.56) \times 10^{-3}[1]$. The two values differ by about 3 standard deviations and for this reason it's important to perform new measurements of $\left|V_{\mathrm{cb}}\right|$ in order to confirm or disprove this apparent incompatibility of the two results.

The exclusive determinations of $\left|V_{\mathrm{cb}}\right|$ rely on the knowledge of the form factors, which are functions of the squared mass of the virtual $W^{+}$emitted in the $\bar{b} \rightarrow \bar{c}$ transition $\left(q^{2}\right)$ and parametrise the strong-interaction in the hadronic current of the quarks bound in mesons. Several parameterisations have been proposed to model the form factors. The one from Caprini, Lellouch and Neubert (CLN) [2] has been extensively used in all the exclusive determinations of $\left|V_{\mathrm{cb}}\right|$ and the approximations contained in this parameterisation have been considered as a possible source of the discrepancy with the inclusive measurement [3-6]. An alternative model has been developed by Boyd, Grinstein and Lebed (BGL) [7-9] and has been used in some recent measurements. As of today, the $\left|V_{\mathrm{cb}}\right|$ values measured in the two parameterisations are not significantly different and the puzzle remains open.

\section{Measurement of $\left|V_{\mathrm{cb}}\right|$ with $B_{s}^{0} \rightarrow D_{s}^{(*)-} \mu^{+} v_{\mu}$ decays}

All exclusive measurements of $\left|V_{\mathrm{cb}}\right|$ performed so far have exploited $B^{0}$ and $B^{+}$mesons. The analysis of other $b$-hadron decays could therefore shed some light on the $\left|V_{\mathrm{cb}}\right|$ puzzle. Semileptonic $B_{s}^{0}$ decays are abundantly produced at the LHC and are more advantageous from a theoretical point of view with respect to the $B^{0}$ and $B^{+}$counterparts, since the form factors in $B_{s}^{0} \rightarrow D_{s}^{-}$transitions are faster to compute and more accurately determined by lattice QCD.

The LHCb collaboration has performed an analysis of $B_{s}^{0} \rightarrow D_{s}^{-} \mu^{+} v_{\mu}$ and $B_{s}^{0} \rightarrow D_{s}^{*-} \mu^{+} v_{\mu}$ decays, using data recorded in proton-proton collisions collected at centre-of-mass energies of 7 and $8 \mathrm{TeV}$ and corresponding to an integrated luminosity of $3 \mathrm{fb}^{-1}$ [10]. In both cases, the $D_{s}^{-} \mu^{+}$ final state is reconstructed, using the Cabibbo-favored mode $D_{s}^{-} \rightarrow K^{+} K^{-} \pi^{-}$. The photon or the neutral pion emitted with the $D_{s}^{-}$in the $D_{s}^{*-}$ decay is not reconstructed.

The value of $\left|V_{\mathrm{cb}}\right|$ is determined from the observed yields of $B_{s}^{0} \rightarrow D_{s}^{(*)-} \mu^{+} v_{\mu}$ decays, using as normalisation channels the $B^{0} \rightarrow D^{(*)-} \mu^{+} v_{\mu}$ decay modes. The ratios of the branching fractions $\mathcal{R} \equiv \mathcal{B}\left(B_{s}^{0} \rightarrow D_{s}^{-} \mu^{+} v_{\mu}\right) / \mathcal{B}\left(B^{0} \rightarrow D^{-} \mu^{+} v_{\mu}\right)$ and $\mathcal{R}^{*} \equiv \mathcal{B}\left(B_{s}^{0} \rightarrow D_{s}^{*-} \mu^{+} v_{\mu}\right) / \mathcal{B}\left(B^{0} \rightarrow D^{*-} \mu^{+} v_{\mu}\right)$ are also measured. Both the CLN and BGL parameterisations are tested.

In this analysis, a novel method is employed for the first time: instead of approximating $q^{2}$, which cannot be precisely determined due to undetected particles in the final state, a variable that can be obtained from reconstructed particles is used. This quantity is the component of the momentum of the $D_{s}^{-}$meson transverse to the $B_{s}^{0}$ flight direction and it is denoted as $p_{\perp}\left(D_{s}^{-}\right)$. This variable is highly 
correlated with the $q^{2}$ of the $B_{s}^{0} \rightarrow D_{s}^{-} \mu^{+} v_{\mu}$ and $B_{s}^{0} \rightarrow D_{s}^{*-} \mu^{+} v_{\mu}$ decays and, to a minor extent, to the helicity angles of the $B_{s}^{0} \rightarrow D_{s}^{*-} \mu^{+} v_{\mu}$ decay, thus being sensitive to the form factors. Also, when used together with the corrected mass $\left(m_{\text {corr }}\right)$, it helps in separating the signal modes from the background. The corrected mass is defined as $m_{\mathrm{corr}}=\left[m^{2}\left(D_{s}^{-} \mu^{+}\right)+p_{\perp}^{2}\left(D_{s}^{-} \mu^{+}\right)\right]^{1 / 2}+p_{\perp}\left(D_{s}^{-} \mu^{+}\right)$, where $m\left(D_{s}^{-} \mu^{+}\right)$is the reconstructed mass of the $D_{s}^{-} \mu^{+}$system and $p_{\perp}\left(D_{s}^{-} \mu^{+}\right)$is the component of the $D_{s}^{-} \mu^{+}$momentum transverse to the $B_{s}^{0}$ flight direction.

The selection requires a high transverse-momentum muon candidate associate with one, two, or three charged particles, all originating from a vertex displaced from the $p p$ interaction point. The $D$ mesons in signal and normalisation channels are required to have an invariant mass in the vicinity of their known masses and the $K^{+} K^{-}$mass to lie close to the $\phi(1020)$ mass. A requirement in the two dimensional $\left(m_{\text {corr }}, p_{\perp}\left(D_{s}^{-}\right)\right)$plane is placed to reject most of the partially reconstructed $b$-hadron decays.

A least-squares fit to the $\left(m_{\text {corr }}, p_{\perp}\left(D_{s}^{-}\right)\right)$distribution of the selected inclusive samples of $D_{(s)}^{-} \mu^{+}$candidates is used to simultaneously determine the form factors and the signal yields needed for the measurement of $\left|V_{\mathrm{cb}}\right|$ or of the ratios of branching fractions $\mathcal{R}^{(*)}$. The shape of each component is modelled with two-dimensional histogram templates derived from either simulation or data candidates. The $m_{\text {corr }}$ and $p_{\perp}\left(D_{s}^{-}\right)$distributions of the signal channels are shown in Fig. 1, with the fit projections overlaid for the CLN parameterisation. The fit performed with the BGL parameterisation yields very similar results.
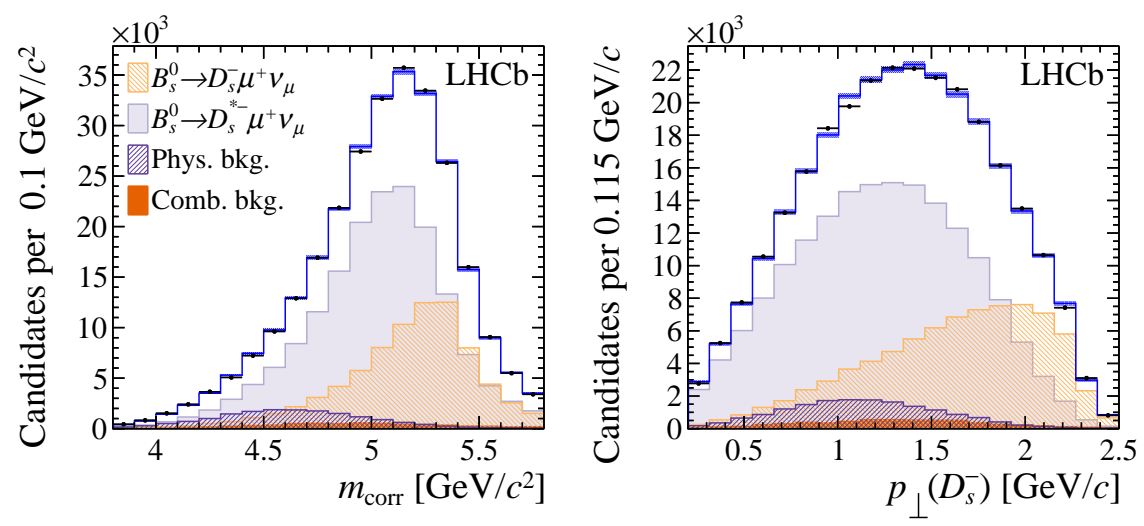

Figure 1: Distribution of (left) $m_{\text {corr }}$ and (right) $p_{\perp}\left(D_{s}^{-}\right)$for $D_{s}^{-} \mu^{+}$decays. Fit projections for the CLN parameterisation are overlaid.

Several sources of systematic uncertainties have been evaluated. The dominant systematic uncertainties on $\left|V_{\mathrm{cb}}\right|$ and $\mathcal{R}^{(*)}$ are due to the knowledge of the $D_{(s)}^{-} \rightarrow K^{+} K^{-} \pi^{-}$model used to generate simulated decays and the ratio of $B_{s}^{0}$-to- $B^{0}$ production fractions taken as external input. The former is estimated by comparing the efficiency of the requirement on the $K^{+} K^{-}$mass between simulation and an independent set of data.

In conclusion, a novel analysis method is used to identify the two exclusive decays $B_{s}^{0} \rightarrow D_{s}^{-} \mu^{+} v_{\mu}$ and $B_{s}^{0} \rightarrow D_{s}^{*-} \mu^{+} v_{\mu}$ and to measure the CKM matrix element $\left|V_{\mathrm{cb}}\right|$ using $B^{0} \rightarrow D^{-} \mu^{+} v_{\mu}$ and $B^{0} \rightarrow D^{*-} \mu^{+} v_{\mu}$ decays as normalisation. The measurement is performed with both the CLN and 
BGL parameterisations and the values of $\left|V_{\mathrm{cb}}\right|$ are found to be

$$
\begin{aligned}
& \left|V_{\mathrm{cb}}\right|_{\mathrm{CLN}}=(41.4 \pm 0.6 \pm 0.9 \pm 1.2) \times 10^{-3} \\
& \left|V_{\mathrm{cb}}\right|_{\mathrm{BGL}}=(42.3 \pm 0.8 \pm 0.9 \pm 1.2) \times 10^{-3}
\end{aligned}
$$

where the first uncertainties are statistical (including contributions from both data and simulation), the second systematic and the third due to external inputs.

The ratios of branching fractions of the two exclusive decays $B_{s}^{0} \rightarrow D_{s}^{-} \mu^{+} v_{\mu}$ and $B_{s}^{0} \rightarrow D_{s}^{*-} \mu^{+} v_{\mu}$ relative to those of exclusive $B^{0} \rightarrow D^{-} \mu^{+} v_{\mu}$ and $B^{0} \rightarrow D^{*-} \mu^{+} v_{\mu}$ decays are also measured to be

$$
\begin{aligned}
\mathcal{R} & =1.09 \pm 0.05 \pm 0.06 \pm 0.05 \\
\mathcal{R}^{*} & =1.06 \pm 0.05 \pm 0.07 \pm 0.05
\end{aligned}
$$

where the first uncertainties are statistical, the second systematic and the third due to external inputs.

Taking the measured values of the branching ratios of $B^{0} \rightarrow D^{-} \mu^{+} v_{\mu}$ and $B^{0} \rightarrow D^{*-} \mu^{+} v_{\mu}$ decays as external input, the following exclusive branching fractions are measured for the first time

$$
\begin{aligned}
\mathcal{B}\left(B_{s}^{0} \rightarrow D_{s}^{-} \mu^{+} v_{\mu}\right) & =(2.49 \pm 0.12 \pm 0.14 \pm 0.16) \times 10^{-2} \\
\mathcal{B}\left(B_{s}^{0} \rightarrow D_{s}^{*-} \mu^{+} v_{\mu}\right) & =(5.38 \pm 0.25 \pm 0.46 \pm 0.30) \times 10^{-2}
\end{aligned}
$$

where the first uncertainties are statistical, the second systematic and the third due to external inputs.

\section{Measurement of the shape of the $B_{s}^{0} \rightarrow D_{s}^{*-} \mu^{+} v_{\mu}$ differential decay rate}

The determination of the form factors in $B_{s}^{0} \rightarrow D_{s}^{*-} \mu^{+} v_{\mu}$ decays obtained in different parameterisations can help to clarify the inconsistency between the exclusive and inclusive determinations of $\left|V_{\mathrm{cb}}\right|$. It can also be used to improve the Standard Model predictions for the $B_{s}^{0} \rightarrow D_{s}^{*-} \tau^{+} \nu_{\tau}$ mode and for the ratio $\mathcal{R}\left(D_{s}^{*-}\right)=\mathcal{B}\left(B_{s}^{0} \rightarrow D_{s}^{*-} \tau^{+} v_{\tau}\right) / \mathcal{B}\left(B_{s}^{0} \rightarrow D_{s}^{*-} \mu^{+} v_{\mu}\right)$. In this analysis, the first measurement of the shape of the differential decay rate of $B_{s}^{0} \rightarrow D_{s}^{*-} \mu^{+} v_{\mu}$ as a function of $w$, where $w \equiv\left(m_{B_{s}^{0}}^{2}+m_{D_{s}^{*-}}^{2}-q^{2}\right) /\left(2 m_{B_{s}^{0}} m_{D_{s}^{*-}}\right)$, is obtained [11]. Then, this shape is fitted with the CLN [2] and BGL [7-9] parameterisations to determine the leading form factors parameters in both cases. The dataset used corresponds to an integrated luminosity of $1.7 \mathrm{fb}^{-1}$ collected by the LHCb experiment in 2016 at a centre-of-mass energy of $13 \mathrm{TeV}$.

Candidate signal events are reconstructed by combining $D_{s}^{*-}$ and $\mu^{+}$candidates. The $D_{s}^{*-}$ meson is reconstructed in the $D_{s}^{-} \gamma$ mode. The $D_{s}^{-}$mesons are formed by requiring two oppositely charge kaons and a pion to form a vertex inconsistent with any primary vertex. Only the $\phi \pi^{-}$and $K^{* 0} K^{-}$regions are selected in the Dalitz plane in order to suppress combinatorial background.

A fit to the $D_{s}^{-} \gamma$ invariant-mass distribution, with the $D_{s}^{-}$mass constrained to its known value, is performed to subtract the combinatorial background and to select candidates that will be used in the remainder of the analysis. Extended binned maximum-likelihood fits are performed to the $m_{\text {corr }}$ distribution in seven bins of the reconstructed hadronic recoil $w$ to obtain the raw signal yields used to build the shape of the differential decay rate. To obtain the value of $w$ one needs to know the $q^{2}$ of the decay and this is determined by using a multivariate regression algorithm based on the flight direction of the $B_{s}^{0}$ meson [12]. 


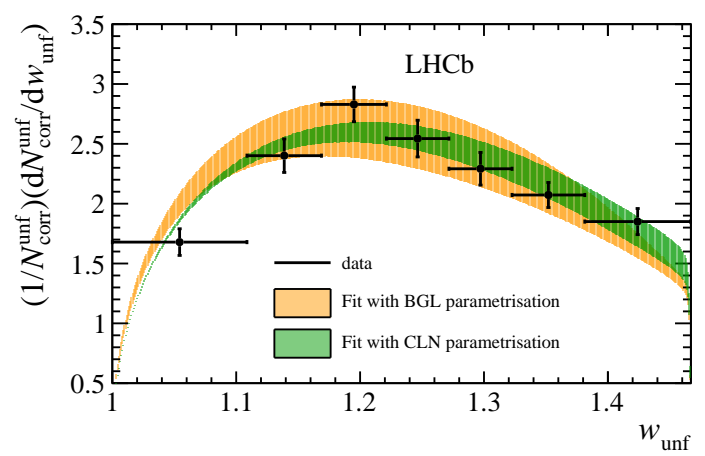

Figure 2: Unfolded and normalised differential decay rate for $B_{s}^{0} \rightarrow D_{s}^{*-} \mu^{+} v_{\mu}$ decays. The green and yellow shaded areas correspond to the CLN and BGL fit projections, respectively.

The measured $B_{s}^{0} \rightarrow D_{s}^{*-} \mu^{+} v_{\mu}$ spectrum needs then to be unfolded to account for the resolution and to be corrected by using the efficiencies determined from the simulated samples. The unfolded, efficiency-corrected and normalised differential decay rate is shown in Fig. 2, with the fit projections obtained for the CLN and BGL parameterisation overlaid. As can be seen, both models provide a good description of the data points.

Several sources of systematic uncertainties are considered. The dominant ones are due to the limited size of the simulated samples, to the knowledge of the efficiencies and to the finite amount of data used to correct the simulated events.

In conclusion, a measurement of the shape of the differential decay rate and of the leading form factors parameters of the $B_{s}^{0} \rightarrow D_{s}^{*-} \mu^{+} v_{\mu}$ decay has been performed. Using the CLN parameterisation the value obtained for the leading form factor parameter is

$$
\rho^{2}=1.16 \pm 0.05 \pm 0.07
$$

where the first uncertainty is statistical and the second systematic.

Using the BGL parameterisation, the results obtained are

$$
\begin{aligned}
& a_{1}^{f}=-0.002 \pm 0.034 \pm 0.046 \\
& a_{2}^{f}=0.93_{-0.20-0.38}^{+0.05+0.06}
\end{aligned}
$$

where the first uncertainties are statistical and the second systematic.

\section{Conclusions}

In these proceedings, the latest results obtained by the LHCb collaboration in the determination of CKM matrix element $\left|V_{\mathrm{cb}}\right|$ have been presented. In particular, the LHCb collaboration measured for the first time $\left|V_{\mathrm{cb}}\right|$ using $B_{s}^{0} \rightarrow D_{s}^{-} \mu^{+} v_{\mu}$ and $B_{s}^{0} \rightarrow D_{s}^{*-} \mu^{+} v_{\mu}$ decays and the form factors of these decay modes using Run 1 data. The results obtained from using both CNL and BGL parameterisation are consistent. As a result, the tension in exclusive and inclusive determination of $\left|V_{\mathrm{cb}}\right|$ cannot solely be attributed to the approximations made in the CNL parameterisation. The 
techniques and methodologies developed in this analysis can also be used with semileptonic $B^{0}$ decays at $\mathrm{LHCb}$ to reach the ultimate precision on the determination of $\left|V_{\mathrm{cb}}\right|$.

The LHCb collaboration also published the shape of the differential decay rate of $B_{s}^{0} \rightarrow D_{s}^{*-} \mu^{+} v_{\mu}$ decays and provided another independent determination of the form factors of this mode using part of Run 2 data. This represents a very useful input for theorist trying to develop alternative parameterisations for the form factors or corrections to the already existing ones.

The results presented in these proceedings prove the capabilities of the LHCb experiment with semileptonic $B_{s}^{0}$ decays and many more important results are expected to come in the next years.

\section{References}

[1] Y. Amhis et al. (Heavy Flavor Averaging Group), Averages of b-hadron, c-hadron, and $\tau$ lepton properties as of 2018, [hep-ex/1909.12524].

[2] I. Caprini, L. Lellouch and M. Neubert, Dispersive bounds on the shape of anti-B $\rightarrow D(*)$ lepton anti-neutrino form-factors, Nucl. Phys. B 530 (1998) 153-181, [hep-ph/9712417].

[3] D. Bigi, P. Gambino and S. Schacht, A fresh look at the determination of $\left|V_{c b}\right|$ from $B \rightarrow D^{*} \ell v$, Phys. Lett. B 769, (2017) 441-445, [hep-ph/1703.06124].

[4] B. Grinstein and A. Kobach, Model-Independent Extraction of $\left|V_{c b}\right|$ from $\bar{B} \rightarrow D^{*} \ell \bar{v}$, Phys. Lett. B 771 (2017) 359-364, [hep-ph/1703.08170].

[5] S. Jaiswal, S. Nandi and S. K. Patra, Extraction of $\left|V_{c b}\right|$ from $B \rightarrow D^{(*)} \ell v_{\ell}$ and the Standard Model predictions of $R\left(D^{(*)}\right)$, JHEP 12 (2017) 060, [hep-ph/1707.09977].

[6] P. Colangelo and F. De Fazio, Scrutinizing $\bar{B} \rightarrow D^{*}(D \pi) \ell^{-} \bar{v}_{\ell}$ and $\bar{B} \rightarrow D^{*}(D \gamma) \ell^{-} \bar{v}_{\ell}$ in search of new physics footprints, JHEP 06 (2018) 082, [hep-ph/1801.10468].

[7] C. G. Boyd, B. Grinstein and R. F. Lebed, Constraints on form-factors for exclusive semileptonic heavy to light meson decays, Phys. Rev. Lett. 74 (1995) 4603-4606, [hep-ph/9412324].

[8] C. G. Boyd, B. Grinstein and R. F. Lebed, Model independent determinations of anti-B $\rightarrow D$ (lepton), $D^{*}$ (lepton) anti-neutrino form-factors, Nucl. Phys. B 461 (1996) 493511, [hep-ph/9508211].

[9] C. G. Boyd, B. Grinstein and R. F. Lebed, Precision corrections to dispersive bounds on form-factors, Phys. Rev. D 56 (1997) 6895-6911, [hep-ph/9705252].

[10] R. Aaij et al. (LHCb collaboration), Measurement of $\left|V_{c b}\right|$ with $B_{s}^{0} \rightarrow D_{s}^{(*)-} \mu^{+} v_{\mu}$ decays, Phys. Rev. D 101 (2020) no.7, 072004, [arXiv:2001.03225 [hep-ex]].

[11] R. Aaij et al. (LHCb Collaboration), Measurement of the shape of the $B_{s}^{0} \rightarrow D_{s}^{*-} \mu^{+} v_{\mu}$ differential decay rate, arXiv:2003.08453 [hep-ex].

[12] G. Ciezarek, A. Lupato, M. Rotondo and M. Vesterinen, Reconstruction of semileptonically decaying beauty hadrons produced in high energy pp collisions, JHEP 02 (2017) 021, [arXiv:1611.08522 [hep-ex]]. 